\title{
Minimizing Energy Consumption in Cloud Datacenters using Task Consolidation
}

\author{
Madhu B.R. ${ }^{\# 1}$, A.S. Manjunatha ${ }^{* 2}$, Prakash Chandra ${ }^{\# 3}$, Chidananda Murthy $\mathrm{P}^{\# 4}$ \\ ${ }^{\# 1}$ Research Scholar, Jain University, Bengaluru, Karnataka, India. \\ br.madhu@jainuniversity.ac.in \\ ${ }^{* 2}$ Manvish eTech Pvt. Ltd., Bengaluru, Karnataka, India. \\ asmanju@manvish.com \\ ${ }^{\# 3}$ M.Tech Student, SET, Jain University, Bengaluru, Karnataka, India. \\ infymee@gmail.com \\ ${ }^{\# 4}$ Research Scholar, Jain University, Bengaluru, Karnataka, India. \\ chidananda.murthy@gmail.com
}

\begin{abstract}
Cloud service providers are experiencing the huge demand for the computational power as most of the companies are outsourcing their IT services to the cloud. This increased popularity of cloud computing has resulted in the establishment of large-scale datacenters with the huge number of computational intensive servers. These datacenters are very much expensive to maintain as they consume enormous amount of electrical energy, which results in the emission of green house gases like carbon dioxide. One of the main reasons for the huge power consumption is the inefficiency of the existing resource scheduling algorithm, which utilizes the existing resources in the non-power aware manner. This paper is based on the idea that the power consumption is directly proportional to the CPU utilization of the server, and hence proposes the task consolidation technique which maps the users requests i.e. task to the virtual machine in such a way that it maximizes the CPU utilization and minimizes the energy consumption at the same time. CloudSim is used as the simulation tool kit to show how the proposed task scheduling technique is more power efficient compared to the existing task scheduling technique.
\end{abstract}

Keyword- Cloud computing, Task consolidation, CloudSim, Energy consumption, Green computing

\section{INTRODUCTION}

The recent trends in the adoption of cloud computing by the companies for the computing services and solutions has increased exponentially due to the various cost cutting features of cloud like flexibility, scalability, pay as you go etc. These trends have increased the demand for the more computational power, which leads to the establishment of more datacenters. The operational cost of cloud datacenters are extremely high as it mainly consists of large number of high performance physical servers and the cooling systems which consumes enormous amount of electrical energy and thus produces huge amount of green house gases like carbon dioxide.

According to the recent survey, the datacenters consume up to $3 \%$ of global electricity production thus emitting 200 million metric tons of carbon dioxide in to the environment. Out of the total electricity consumed by these datacenters only $10-15 \%$ of the power is used for the actual computation purpose while rest is wasted due to the various inefficiencies existing in the datacenters. The above-mentioned statistics clearly states that there is huge scope for the improvement of the power efficiency and move toward the green computing.

The virtualization layer, which uses the virtualization technology, enables the sharing of single physical server among multiple users by creating number of virtual machines (VMs), which are mapped to the single server. The cloud service providers provide the computational resources in the form of virtual machines to the cloud users. These VMs are responsible for executing the requests of the cloud users. Since the energy consumption is directly related to the CPU utilization, the efficiency of any power aware algorithm greatly depends upon the VM scheduling policy adopted at both the host (server) and task level. This paper presents the task consolidation technique, which assigns multiple tasks to single virtual machine depending upon its processing capability measured in terms of MIPS (Million Instructions Per Second). 


\section{RELATED WORK}

Due to the increasing adoption of cloud computing for all types of IT services and solutions, there has been a lot of research work going on to increase the efficiency of the cloud computing system. As cloud datacenter are becoming the major source of environmental pollutions and very expensive to maintain, there has been continuous research efforts to make the cloud datacenter energy-performance efficient. A novel genetic algorithm has been proposed in [2] for the task scheduling and to improve the energy consumption in cloud systems.

They have used the house simulator to compare the energy efficiency of proposed technique with the random and round robin approach used for the task scheduling. The authors have analyzed various existing energy conscious task consolidation techniques like: ECTC (Energy conscious task consolidation), MAXUtil (Maximum rate utilization) and bi-objective task consolidation technique [4]. In order to minimize the make span time, operational cost and to focus on green computing, they have proposed energy-aware task consolidation technique using the genetic algorithm [5]. The energy aware task consolidation (ETC) is proposed in [6], which minimizes the energy consumption by consolidating the task among the virtual clusters and restricting the CPU usage below specified threshold. They have proposed energy aware. task consolidation (EATC), which considers various factors such as description of physical hosts, virtual machines and task that affect the energy consumption in cloud datacenters [7].

\section{EXISTING PROBLEM}

The physical computing servers at the cloud datacenters are never idle, most of the time they operate at $10-15 \%$ of their full capacity and their utilization rarely reaches $100 \%$. The problem with this utilization model is that even when the servers are in idle state they still consume $70 \%$ of their peak power. Adopting the following approach can prevent this energy inefficiency of the datacenter by:

i) leveraging the virtualization technology which creates multiple virtual machines on the single physical server thus by maximizing the utilization of the resources.

ii) using the live virtual machine migration which consolidates the VMs to the less number of servers depending upon their current resource usage pattern and switching the remaining idle servers to the less power consuming modes such as sleep or hibernate.

The major problem with the live VM migration is that the resource usage pattern of the modern service application can't be predicted and hence frequent live migration of virtual machines can lead to performance degradation and the service level agreement signed between the consumer and cloud provider can be violated. The above-mentioned problems have clearly stated the need of efficient resource scheduling algorithm which can:

i) efficiently allocate the virtual machines to the available servers.

ii) execute the users request i.e. task to the appropriate virtual machine, by maintaining the energyperformance trade-off in the cloud computing system [1].

\section{PROPOSED SOLUTION}

\section{A. System model}

The cloud datacenter consists of number of physical computing server also known as hosts. Each host has their pre-configured processing capability (expressed in MIPS), storage, memory and the provisioning policy to assign the processing cores to the virtual machines. The hosts are assigned to one or more virtual machines (VMs), and these virtual machines are provisioned with the user's application services using the application provision policy and are responsible for executing the users request in the form of cloudlets.

The energy efficiency of any cloud computing system depends upon how efficient resource provisioning policy it uses. In cloud the resource provisioning policy are defined at two levels: at host level and at VM level. At the host level the policy is responsible for assigning the overall processing cores to each of the virtual machines and at the VM level the policy assign the fixed amount of the processing capability to each of the tasks i.e. cloudlet hosted within the VM. 


\section{B. Energy model}

The energy model is based on the idea that the power consumption has linear relationship with the CPU utilization. So we have tried to decrease the CPU utilization and the execution time of the cloudlet in order to decrease the power consumption in cloud.

The proposed solution to minimize the energy consumption uses the task consolidation technique. Let us consider the total number of task to be executed is ' $\mathrm{m}$ ' then task consolidation process assigns $\mathrm{n}$ tasks out of $\mathrm{m}$ to the virtual machines by maximizing the resource utilization and minimizing the overall energy consumption.

We have used the CloudSim as the simulation tool kit for the simulation of the proposed task consolidation technique. Following are the configuration for the host and the virtual machines that are used for the simulation:

For the host:

RAM $=10000$, bandwidth $=100000$, storage $=1000000$

MIPS $=1000$

For the virtual machine:

MIPS $=\{250,500,750,1000\}$, Number of $\mathrm{CPU}=1$,

$\mathrm{RAM}=128 \mathrm{MB}$, bandwidth $=2500$, image size $(\mathrm{MB})=2500$

VMM (virtual machine manager) = "XEN".

1) Resource provisioning at the host level: The virtual machine allocation policy uses the technique one VM per host i.e. one virtual machine is allocated to only one host at a time. The pseudo code for the VM allocation policy used is as follows:

Let total number of host is ' $m$ ' and the number of VM that need to be allocated is ' $n$ '. let $i=0, j=0$.

While $(i<m$ and $j<n)$ :

AllocateHostForVM (VM.id (j), Host.id (i))

$\mathrm{i}=\mathrm{i}+1$

$\mathrm{j}=\mathrm{j}+1$

EndWhile

2) Resource provisioning at the VM level: The total processing capability assign to each host is 1000 MIPS while each VMs are assigned with the processing capacity of 250, 500, 750, 1000 MIPS iteratively. Since only one VM is assigned per host, the VM with processing capacity of 250 MIPS can execute 4 tasks/ cloudlets at a time, the VM with the capacity of 500 MIPS can execute 2 cloudlets, the VM with capacity of 750 and 1000 MIPS can execute only one cloudlet at a time. We have used the above-mentioned concept to consolidate cloudlets to the VMs. In CloudSim the datacenter broker is responsible for assigning the cloudlets to virtual machines. Following is the pseudo code that datacenter broker uses for the task consolidation:

Function submitcloudlet ()

If (VM.getMIPS $==250$ )

Assign 4 cloudlet to the VM

Else if (VM.getMIPS $==500$ )

Assign 2 cloudlet to the VM

Else if (VM.getMIPS==750)

Assign 1 cloudlet to the VM

Else

Assign 1 cloudlet to VM 


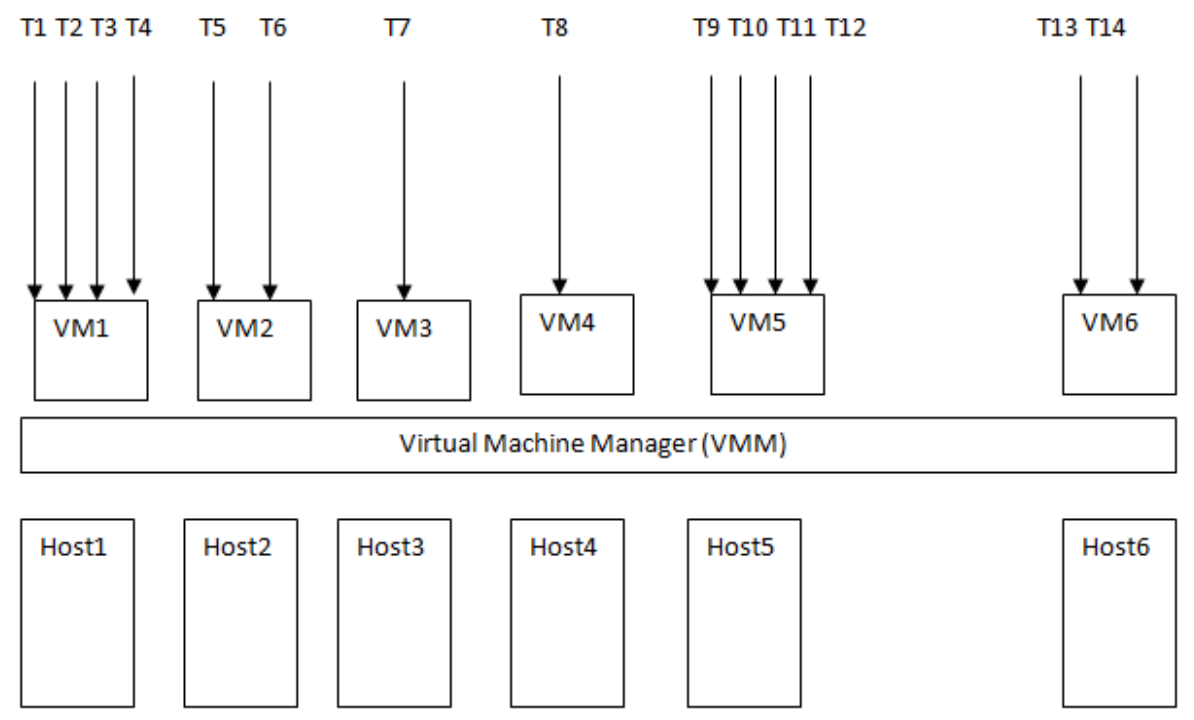

Fig 1: Architecture diagram of the proposed solution

\section{RESULT ANALYSIS}

For experimentation purpose the simulation was carried out using the CloudSim as the simulation tool kits on Mac OS X with the system configuration of 4GB RAM, 2.5 GHZ Intel core i5 processor. The configuration of hosts and virtual machines used for the simulation has the same configuration as mentioned above. The energy efficiency of the proposed task consolidation technique is compared with the Round Robin used by the CloudSim and the result obtained from the simulation is shown in Table 1.

\begin{tabular}{|l|l|l|}
\hline Cloudlet number & Task consolidation & Round Robin \\
\hline 4 & 0.07 & 0.11 \\
\hline 8 & 0.15 & 0.18 \\
\hline 16 & 0.30 & 0.37 \\
\hline 24 & 0.44 & 0.58 \\
\hline 32 & 0.59 & 0.77 \\
\hline
\end{tabular}

Table 1 : Energy Consumption in Round Robin and Task Consolidation

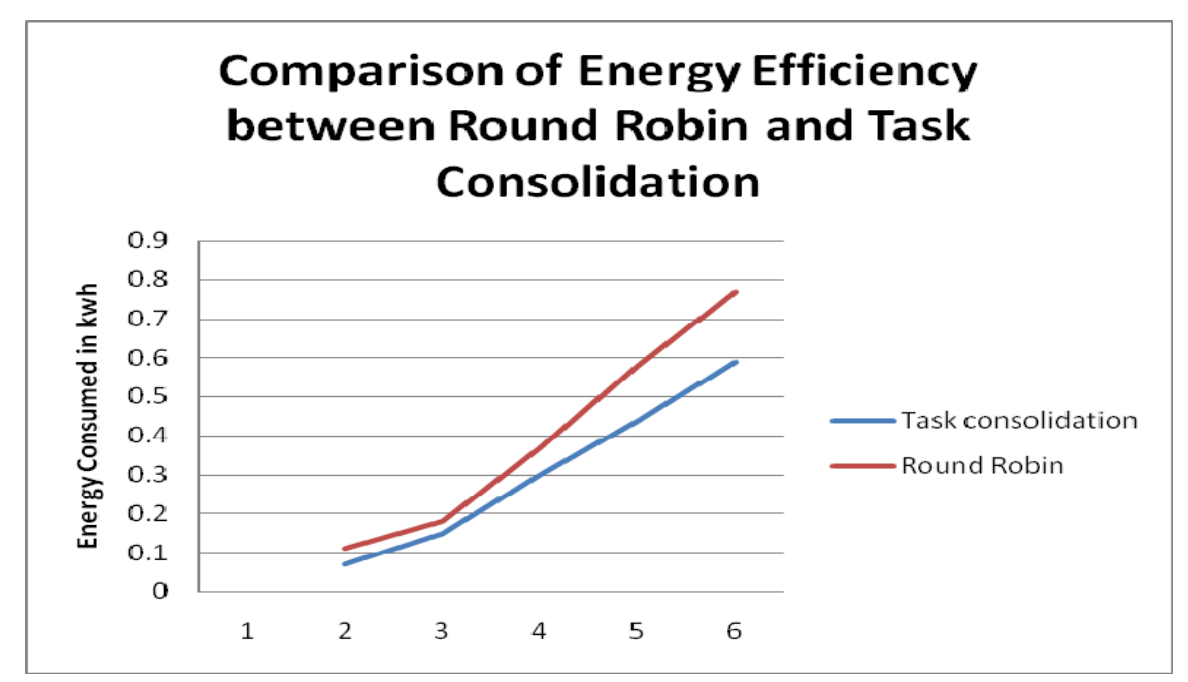

Fig 2 : Comparison of Energy Efficiency between Round Robin and Task Consolidation 
According to paper [1,3] when local linear regression (LRR) technique is combined with the minimum migration time (MMT) as the VM selection policy it outperforms other dynamic VM consolidation technique and achieves much better energy consumption with very less VM need to be migrated. When the above mentioned LRR technique is modified by applying the task consolidation technique, the simulation results shows that the modified LRR technique performs much better compared to the existing LRR technique of CloudSim as shown in Table 2.

\begin{tabular}{|c|c|c|c|c|}
\hline $\begin{array}{l}\text { Number } \\
\text { of } \\
\text { cloudlet }\end{array}$ & \multicolumn{2}{|l|}{ Modified LRR-MMT } & \multicolumn{2}{l|}{ LRR-MMT } \\
\hline & $\begin{array}{c}\text { VM } \\
\text { migrated }\end{array}$ & $\begin{array}{c}\text { Energy } \\
\text { consumed }\end{array}$ & $\begin{array}{c}\text { VM } \\
\text { migrated }\end{array}$ & $\begin{array}{l}\text { Energy } \\
\text { consumed }\end{array}$ \\
\hline 16 & 5 & 0.07 & 13 & 0.10 \\
\hline 24 & 8 & 0.08 & 22 & 0.14 \\
\hline 32 & 14 & 0.12 & 27 & 0.17 \\
\hline
\end{tabular}

Table 2 : Comparison of Energy Efficiency between Round Robin and Task Consolidation

\section{CONCLUSION}

The results show that the efficient task scheduling technique plays very important role in minimizing the energy consumption. The task consolidation approach for scheduling task to the VM compared with the existing round robin approach of the CloudSim, and the derived result clearly shows how the proposed approach outperforms the existing technique. Also, modifications done to the task scheduler of the LRR-MMT technique of CloudSim by using the task consolidation approach, when compared with the results of the original LRRMMT approach of CloudSim, the experiment results shows that the number of VM to be migrated is significantly reduced, which in turn aids to lesser energy consumption in the datacenter.

\section{REFERENCES}

[1] Anton Beloglazov and Rajkumar Buyya . "Optimal Online Deterministic Algorithms and Adaptive Heuristics for Energy and Performance Efficient Dynamic Consolidation of Virtual Machines in Cloud Data Centers ". Published online in Wiley InterScience www.interscience.wiley.com

[2] Dilip Kumar,Bibhudatta Sahoo,Bhaskar Mondal,Tarni Mandal.“A Genetic Algorithmic approach for Energy Efficient Task Consolidation in Cloud Computing ". International Journal of Computer Applications (0975 8887) Volume 118 - No. 2, May 2015.

[3] Madhu B. R. ,Dr A.S. Manjunatha, Prakash Chandra ,Chidananda Murthy P. . "A Comparative Study of Algorithms For Efficient Dynamic Consolidation of Virtual Machines In Cloud ". International Journal of Applied Engineering Research ISSN 0973-4562 Volume 11, Number 6 (2016) pp 4597-4600

[4] Amandeep kaur ,Rupinder kaur ,Prince Jain ."Algorithms for Task Consolidation Problem in a Cloud Computing Environment ". International Journal of Computer Applications (0975 - 8887) Volume 75- No.4, August 2013

[5] Ipsita Kar, R.N. Ramakant Parida ,Himansu Das. "Energy Aware Scheduling using Genetic Algorithm in Cloud Data Centers ". International Conference on Electrical, Electronics, and Optimization Techniques (ICEEOT) - 2016

[6] Ching-Hsien Hsu, Kenn D. Slagter, Shih-Chang Chen, Yeh-Ching Chung. "Optimizing energy consumption with task consolidation in clouds ". Information Sciences 258 (2014) 452- 462.

[7] Sambit Kumar Mishra, Reenu Deswal, Sampa Sahoo, Bibhudatta Sahoo "Improving Energy Consumption in Cloud", Annual IEEE India Conference (INDICON), 2015. 